J. Lake Sci. (湖泊科学) , 2015, 27(1): 1-10

http://www. jlakes. org. E-mail : jlakes@niglas.ac.cn

(c) 2015 by Journal of Lake Sciences

\title{
全球变暖对淡水湖泊浮游植物影响研究进展
}

\author{
邓建明 ${ }^{1,2}$, 秦伯强 ${ }^{1 * *}$ \\ (1:中国科学院南京地理与湖泊研究所湖泊与环境国家重点实验室, 南京 210008) \\ (2: 中国科学院大学,北京 100049 )
}

\begin{abstract}
摘 要: 全球变暖对湖泊生态系统的影响已经成为近年来湖沼学领域的研究热点. 本文首先列举了目前研究全球变暖 对淡水湖泊浮游植物影响的常用方法:监测数据分析、时空转换、遥感信息提取、控制实验、模型预测和古湖沼学技术等. 研究结果表明气候变暖导致的气温升高、湖泊热力分层提前破坏以及无冰期提前等因素可导致春季物候提前; 在全球变 暖大背景下浮游植物群落结构正朝着蓝澡占优的方向发展,但是不同地区以及不同物种对全球变暖的响应不一致. 在营 养盐充足的湖泊中, 由于全球变暖延长了浮游植物生长季节等, 从而能提高浮游植物初级生产力; 但在贫营养湖泊中, 浮 游植物初级生产力与变暖趋势甚至可能呈负相关. 由于生态系统往往是多因子的共同作用, 这也使得全球变暖对浮游植 物群落的影响效应复杂化, 区分各因子的净影响份额是目前研究的一个难点; 全球变暖引起的风场改变会促进浅水湖泊 中营养盐从底泥的释放, 同时也会增加水体中悬浮物的浓度而影响水下光场, 因此开展气候变化对再悬浮及浮游植物群 落结构的影响可能是将来研究的一个切人点.
\end{abstract}

关键词: 气候变化;浮游植物; 春季物候;初级生产力; 蓝藻

\section{A review on studies of effects of climate change on phytoplankton in freshwater systems}

DENG Jianming ${ }^{1,2} \&$ QIN Boqiang ${ }^{1}$

(1: State Key Laboratory of Lake Science and Environment, Nanjing Institute of Geography and Limnology, Chinese Academy of Sciences, Nanjing 210008, P. R. China)

(2: University of Chinese Academy of Sciences, Beijing 100049, P. R. China)

Abstract: The impact of global warming on lake ecosystems has been of central and growing interests to limnologists recently. Here, we describe the strengths and weaknesses of the multi-faceted approaches that are presently available for elucidating the effects of climate change on phytoplankton, including time series, space-for-time substitution, remote sensing, experiments, modeling and palaeolimnology. Phenology has been impacted due to rising temperatures, earlier thermal stratification and the disappearance of ice-covers on some lakes. These changes have greatly favored increasing dominance by cyanobacteria in lakes globally. Species-specific changes have been observed in response to global warming in different regions around the globe. In lakes where nutrient supplies are sufficient, primary production of phytoplankton has been enhanced by global warming. However, primary production might be more inhibited when nutrients are limiting. Because environmental factors invariably interact with each other, knowing how to evaluate the effects of a single factor on phytoplankton composition and activity is the key research challenge. Changes in wind speed and patterns caused by global warming may affect nutrient releasing, as well as the light regimes in the water column in shallow lakes. Therefore studies on wind-driven effects of sediment and nutrient resuspension events induced by global warming on phytoplankton community succession in large shallow lakes are a future research frontier in limnology.

Keywords : Climate change; phytoplankton; phenology; primary production; cyanobacteria

近年来, 国内外关于气候变化的学术活动和报道层出不穷, 探讨气候变化已经成为当今湖沼学研究的

* 中国科学院对外合作重点项目 (GJHZ1214)、国家自然科学基金重点项目 (41230744) 和中国科学院南京地理与湖 泊研究所”一三五” 战略发展规划项目 (NIGLAS2012135003) 联合资助. 2014-04-10 收稿;2014-07-08 收修改 稿. 邓建明(1985 ), 男, 博士研究生;E-mail: qingyi1207@ 126. com.

** 通信作者;E-mail:qinbq@ niglas. ac. cn. 
一个热点. 全球变暖是近 100 年来气候变化的一个主要特征 ${ }^{[1]}$, 它正影响着人类赖以生存的淡水生态系 统 ${ }^{[2]}$. 全球变暖对淡水生态系统的影响主要来自气温、降雨和风场 (风向、风速)的改变. 湖泊生态系统对上 述变化的响应主要包括湖泊的物理特性如热力分层状况、流域水文特征改变或者冰期的变化,此外, 冰期的 改变又将影响水柱化学特性如改变溶解氧浓度、营养盐循环, 甚至可能改变水色 ${ }^{[3]}$. 全球变暖导致的风暴天 气加剧、降雨格局改变、土壤变暖和冰川融化等能改变流域内营养盐向湖泊水体的输人 ${ }^{[4-6]}$, 从而可能加剧 湖泊的富营养化 ${ }^{[7]}$. 全球变暖也可能引起水生生物的分布格局发生变化, 从而引起物种人侵 ${ }^{[8]}$. 另外, 全球 变暖还将改变物候 ${ }^{[9]}$.

浮游植物因其生长周期快, 生物量以及群落结构对环境的变化非常敏感, 因此常被用来研究水生态系 统对环境的响应 ${ }^{[10]}$. 本文以浮游植物为中心, 首先综述了全球变暖对浮游植物影响的主要研究方法及其优 缺点, 然后总结了当前获得的相关结论.

\section{1 全球变暖对湖泊生态系统中浮游植物影响的研究方法}

长期监测是获取数据最直接的方式. 例如: Wagner 等 ${ }^{[11]}$ 分析了德国柏林 Müggelsee 湖 1982-2007 年的 夏季周监测数据, 研究期间夏季出现水体分层现象的频率增加; 但湖水热力学特性没有显著的变化特征. Ventelä 等 ${ }^{[12]}$ 利用皮海湖的监测数据讨论了冬季气候变化趋势对夏季浮游植物的影响, Cai 等 ${ }^{[13]}$ 和 Deng 等 ${ }^{[14]}$ 利用太湖监测数据讨论了春季温度对水体叶绿素浓度以及微囊藻生物量的影响. 许多基于历史监测 数据的研究表明当前的变暖趋势已经影响了深水湖泊的热力学分层 (从而间接影响浮游植物 ${ }^{[15]}$ )、促进了 蓝藻优势 ${ }^{[16]}$ 、延长了生长季节等. 对监测数据的分析能直接反映浮游植物对全球变暖的影响, 同时也存在 着不足. 首先是浮游植物种类鉴定数据前后的一致性较差, 造成这种现象的原因可能是负责藻类鉴定人员 不同 (包括鉴定人员自身鉴定水平的提高) 、浮游植物采样及分析方法发生变化、种类分类依据发生改变 等 ${ }^{[17]}$, 因此我们在讨论浮游植物演替, 尤其是浮游植物物种数及多样性时应考虑上述因素的影响. 其次, 湖 泊的监测时间不会太长 (一般 $30 \sim 40$ 年), 并且开展监测的目的通常不是研究气候变化对浮游植物的影响 (往往是富营养化、酸化等过程的影响) ; 加上各个因子之间本身就存在交互影响, 因此通过长期监测数据很 难将全球变暖对浮游植物的单独影响分离出来 ${ }^{[18]}$.

另外一种研究方式是 “空间换时间”, 这种方法的特点是沿着气候梯度选择研究区域, 从而反映在不同 气候下浮游植物的差异. 例如 Kosten 等 ${ }^{[19]}$ 采用了 “空间换时间” 的方式, 选择了分布于从欧洲到南美洲的 143 个不同纬度的湖泊研究了营养盐与水温对浮游植物的影响. 作者试图通过不同纬度的气候特点来探讨 气候变暖后湖泊生态系统的变化趋势. Rigosi 等 ${ }^{[20]}$ 利用美国 > 1000 个湖泊 2007 年的一次调查数据分析了 营养盐浓度和温度对蓝藻的影响. “空间换时间” 的方法在一定程度上能弥补缺乏长时间监测的不足, 但这 种方法的缺陷是很难在不同纬度带选择其他条件一致 (如湖泊形态、营养盐水平、水利条件等) 的湖泊作为 研究对象, 因此得出的结论往往是基于推测 ${ }^{[18]}$.

最近得到广泛使用的另一种研究气候变化对浮游植物影响的手段是遥感反演. 相对于传统依靠采样船 的监测, 卫星携带的探头能定期提供大范围的卫星影像. 通过卫星影像, 我们能用相对较低的成本来获得水 体表面叶绿素时空分布格局的信息. 通过高分辨率的卫星影像 (主要来自 Landsat TM 卫星) 反演水体中叶 绿素浓度在 $1990 \mathrm{~s}$ 初期便引起了科学家的兴趣 ${ }^{[21-22]}$. 后来随着卫星技术的发展, 特别是搭载 MODIS (Moderate-resolution Imaging Spectroradiometer) 的 Terra 和 Aqua 两颗卫星成功投人使用,使我们能获得的卫星影 像的数量以及频次越来越多. Duan 等 ${ }^{[23]}$ 和 Zhang 等 ${ }^{[24]}$ 主要利用卫星影像的数据, 反演了 1987-2009 年的 太湖水华事件的首次暴发日期和持续时间. 遥感技术的运用增加了获得历史资料的可能性, 同时能提供频 率更高的数据; 由于遥感卫星图片可以免费获取, 因此其研究成本也比较低. 但是由于遥感分辨率以及天气 影响的原因, 使得它的运用也受到限制 ${ }^{[23]}$; 同时目前遥感还只能用来监测表层水华, 不能获得种类组成等 信息.

此外, 控制实验也是使用较多的一种研究方法. 近年来通过模拟增温过程来研究湖泊系统对气候变暖 响应的报道越来越多, 实验设计也层出不穷. 因为气候变化引起的增温幅度往往较小, 因此本文不考虑生理 实验中常见的温度梯度实验. McKee 等 ${ }^{[25]}$ 报道了一套大规模的实验装置, 作者使用 48 个 $3200 \mathrm{~L}$ 的桶为实 
验容器, 这些桶埋在利物浦附近的 Ness 植物园, 并通过热交换的方式保证加热处理中水温持续高于环境温 度 $\left(3^{\circ} \mathrm{C}\right)$. 该装置温控效果尚可,但由于蒸发作用显著, 因此需要定期补充去离子水. Moss 等 ${ }^{[26]}$ 在 1998 年 9 月-2000 年 9 月期间使用这套装置研究了气候变暖、营养盐浓度和鱼类对浮游植物的影响. Liboriussen 等 ${ }^{[27]}$ 在 $\mathrm{McKe}$ 等 ${ }^{[25]}$ 的实验装置上有所改进: 增加了流动加水 (地下水) 系统, 使得这个系统能克服由于蒸 发带来的损失. 这个装置自 2003 年开始运行, 并持续运行至今. Yvon-Durocher 等 ${ }^{[28]}$ 在英国也设计了类似 的实验: 2005 年 12 月一 2008 年 4 月间布置了 20 个人工小池塘, 每个能容纳 $1 \mathrm{~m}^{3}$ 的水. 其中 10 个通过电极 加热, 维持温度比环境温度高 $4^{\circ} \mathrm{C}$. 类似大规模实验需要较大的人力和物力投人, 因而开展的更广泛的受控 实验是小规模实验. de Senerpont Domis 等 ${ }^{[29]}$ 在 Tjeukemeer 湖 (位于荷兰北部) 利用 12 个 $10 \mathrm{~L}$ 的容器研究 了 3 组不同温度条件下的浮游植物群落结构. 整个实验采用半连续培养的装置持续了 $61 \mathrm{~d}$, 利用精度较高 的温控仪控制每天的温度并逐日递增 (起始温度都是 $9^{\circ} \mathrm{C}, 3$ 个不同处理最后增温分别是 $13 、 19$ 和 $25^{\circ} \mathrm{C}$, 模 拟的时段是该地区春季水华发生时期). Zhang 等 ${ }^{[30]}$ 认为全球变暖不仅仅意味着平均温度的升高,其同时也 使得短期的温度波动更加剧烈和频繁, 因此,评估全球变暖对水华蓝藻种群优势形成的影响要考虑短期温 度波动. 为此他们设计了两组增温实验. 在 $250 \mathrm{ml}$ 的容器内混合培养纯种的微囊藻和小球藻, 所有实验都 是从 $12^{\circ} \mathrm{C}$ 开始. 第 1 组实验每天 $10: 00$ 增加 $3^{\circ} \mathrm{C}$, 对照组为温度持续恒定在 $12 、 15 、 18 、 21 、 24 、 27 、 30$ 和 $33^{\circ} \mathrm{C}$. 第 2 组实验分 3 个处理, 每个处理在实验开始后 3 小时内增温幅度分别是 $3 、 6 、 9^{\circ} \mathrm{C}, 3$ 个对照组每隔 $1 \mathrm{~h}$ 分别增温 $1 、 2 、 3^{\circ} \mathrm{C}$, 直到最后温度为 $15 、 18 、 21^{\circ} \mathrm{C}$.

控制实验的优势在于能分析单一因子对浮游植物的影响 ${ }^{[27]}$, 这对于我们从机理上认识浮游植物对全球 变暖的响应是非常有帮助的 ${ }^{[18]}$. 但是在野外实际情况中,许多环境因子之间还存在交互作用, 因此控制实 验的结果在外推到野外实际情况时常常有偏差. 同时限于条件,在许多控制实验中仅仅控制平均温度单个 因素,而不考虑其他诸如降雨、极端天气现象以及温度波动等因素 ${ }^{[31-32]}$.

通过模型估计来预测未来气候变化情景下浮游植物的变化也是常用的方法之一. 模型的应用在过去的 30 年间得到了迅速的发展; 如今, 各种理化条件综合影响的模型已经成为在全球变暖环境下我们认识浮游 植物变化以及预测未来变化趋势的主要工具. 常用的模型有局部气候模型 (Regional Climate Models, RC$\mathrm{Ms})^{[33]}$ : 首先使用某个气候模型来预测未来气候条件, 然后将所得的气候条件作为驱动因子输人另一个包 含浮游植物的湖泊模型, 从而获得在未来气候条件下浮游植物群落的变化. 在 Howard 等 $^{[34]}$ 研究中, 他们首 先使用 HADCM2 模型获得 Farmoor 水库 (UK) 地区未来 90 年的气候变化趋势, 然后将这种变化趋势带人 CLAMM 模型中评估了微囊藻在未来 90 年的变化. 但在他们的研究中, 温度并没有包含在内. Elliott 等 ${ }^{[35]}$ 在对 Bassenthwaite 湖的研究中, 作者先用 RCM 对 HADCM2 的输人结果进行降尺度处理,然后将获得的气候 条件输人 PROTECH 模型来获得浮游植物群落结构的变化. 以上两个研究都只是考虑了气候因素对藻类生 长的直接影响, 另外还有的研究将气候变化对流域的影响也考虑在内, 这种影响主要是营养盐的输人改变. Arheimer 等 ${ }^{[36]}$ 评估了若干小尺度气候情景对 Rönneå 流域及富营养状态 Ringsjön 湖 (瑞典) 的影响. 作者先 用 PROBE ( PROgram for Boundary layers in the Environment) 模拟了在若干种气候情境下湖泊物理参数, 然后 耦合 BIOLA (BIOgeochemical LAke model) 模型对未来蓝藻情形进行预测. 与局地气候模型相对应的还有敏 感系数法 ( sensitivity approach $)^{[33]}$. 该方法使用一个程序来模拟当前湖泊系统, 然后改变程序里面例如温 度、营养盐等一个或两个参数重新运行. 这种方法允许同时研究两个驱动因子, 还能识别非线性变化和获得 引起变化的阈值. Elliott 等 ${ }^{[37]}$ 首次用敏感系数这种方法采用 PROTECH 模型模拟了营养盐输人与水温对 Bassenthwaite 湖浮游植物的共同影响.

然而, 模型分析结果是否可靠, 首先依赖于我们对模型背后生态过程机理的认识程度 ${ }^{[38]}$; 其次由于缺少 高质量的数据率定模型也使模型运用面临着巨大挑战 ${ }^{[18]}$. 对于缺少高质量数据的限制, Nielsen ${ }^{[39]}$ 提供了一 种新的解决思路——组合多个参数数据集, 这或许能促进模型的进一步发展.

古湖沼学是另外一种历史数据获取办法, 主要是根据湖泊沉积物中残留的硅藻或其他藻类的残体进行 分析,重建历史时期湖泊水体中浮游植物的物种组成,结合同期已知的环境条件来探讨环境变化 (含全球变 暖) 对浮游植物的影响. 主要优势有: 第一, 它能获得比较长的历史数据 (百年尺度及以上) 并真实反映湖泊 的发展历程. 同时由于近 200 年来人类活动对湖泊影响强烈 ${ }^{[40]}$, 而古湖沼学获得更为久远的数据 ${ }^{[41]}$ 则有助 
于我们排除人类活动对浮游植物的影响; 第二, 相比于遥感数据, 古湖沼学能获得比较详细的物种组成. Dong 等 ${ }^{[42]}$ 利用古湖沼学分析了 Esthwaite 水域(英国)沉积物中的硅藻组成, 并结合营养盐和水温数据研究 了营养盐以及温度对硅藻群落演替的贡献大小. Thies 等 ${ }^{[43]}$ 利用古湖沼学的方法研究了 19 世纪末至今 Piburger See 环境变化、鉴定并分析了各层样本中的硅藻和藻类色素等信息,通过 DI - TP 转换函数重建了样 本当时的总磷浓度, 结果表明 20 世纪该湖朝着富营养化发展, 1970 年开始的湖泊修复使得 $1980 \mathrm{~s}$ 中期湖泊 有缓慢的好转趋势; 温度数据显示在 $1940 \mathrm{~s}$ 中期开始有上升趋势, 并且在 20 世纪后期开始影响藻类生长和 种类组成. 古湖沼学的方法适用于人类活动干扰少、湖泊沉积稳定的湖泊中, 在浅水或人类活动强烈的湖泊 中的运用可能因为沉积物被破坏而受到影响. 另外, 古湖沼学对于整个浮游植物群落结构的认识也有不足, 因为该方法获得的浮游植物群落信息主要集中在硅藻等.

\section{2 全球变暖对浮游植物影响的主要研究结论}

引起气候变化原因的假说有很多 ${ }^{[1]}$. 目前的气候变暖是近 100 年气候变化的最显著特征, 基本上已经 公认它是由人类活动产生的以 $\mathrm{CO}_{2}$ 为主要代表的温室气体增加所引发的气候效应. 虽然刚过去的 100 年已 经发生的气候变暖幅度还没有超过历史的极值幅度, 但已经引起了全社会的高度警觉. 近 100 年的人类活 动对气候变化较过去有了更大的影响, 这大大改变了气候变化的规律, 反自然的变化趋势十分明显, 增加了 气候变化的不确定性和人为性,增大了预测气候变化的难度.

\section{1 全球变暖使浮游植物春季物候提前}

无论陆地生态系统还是水生生态系统, 世界范围内都有春季物候提前的记录 ${ }^{[44-47]}$. 在淡水湖泊 Müggelsee 中 1979-1998 年间也有浮游植物物候提前的记录 ${ }^{[48]}$. 全球变暖对水生生态系统物候的影响机理 主要有温度 (或者光照 ${ }^{[49]}$ ) 的直接影响和湖泊分层时间、冰期时间缩短的间接影响.

温度升高能直接增强浮游植物光合作用和呼吸作用, 从而提高生长率及繁殖速率 ${ }^{[50-51]}$, 缩短浮游植物 生物量达到峰值的时间使得物候提前. 冬、春季节的优势种一般是对营养盐要求较高的硅藻, 因为它生长速 率快, 同时对光强和温度的要求相比其他藻类低 ${ }^{[52-53]}$, 因此硅藻能以累积生物量的方式快速对水温升高作 出响应. 虽然温度的影响是直接的、显而易见的, 但也有其他研究发现温度往往不是最显著的影响因子. 例 如 Thackeray 等 ${ }^{[54]}$ 在 Windermere 湖的研究认为热力分层是导致 Cyclotella 生物量提前达到峰值的主要因素, 而营养盐浓度对 Asterionella 物候的调节作用要大于水温.

湖泊分层现象的发生是因为水的密度在 $4^{\circ} \mathrm{C}$ 时最大,一般发生在冬春、秋冬季节气温变化时, 在水体中, 特别是温带深水湖泊中. 经过整个冬季, 水柱中水体密度由上至下逐渐增加, 形成稳定的水柱有利于表层漂 浮藻类获得充足的光照; 冬季向春季过渡时, 随着气温逐渐回升, 表层水温开始升高, 表层水密度也开始增 加, 这样密度梯度不再稳定, 从而发生水体的垂直交换; 这种情况下表层的藻类就会被带到水底, 同时也能 给底部补充氧气, 而底部营养盐也能随着上升水流来到表层, 因此这种季节间的水体垂直交换对藻类很关 键 $^{[55]}$. 气候变暖使得春季温度升高提前从而使热力分层现象提前发生 ${ }^{[55]}$, 春季物候也因此而提前. 因此可 以说气候变化引起水体热力分层的变化是气候对浮游植物物候的一种间接影响 ${ }^{[66]}$. 这种影响在深水湖泊 中更加显著.

冰期的改变也能使浮游植物春季物候提前. 冰的存在对浮游植物的影响是多方面的: 冰的厚度影响着 水柱里的光; 同时, 有冰覆盖时, 湖水湍流下降, 形成一个稳定垂直的水温、光照、溶解氧和营养盐浓度. 冰面 上降雪与否以及降雪厚度大小能影响其透光性, 从而影响浮游植物在水柱中的分布及其种类组成 ${ }^{[57]}$. 气候 变化也将引起冰期的改变 (结冰时间以及融化时间 ${ }^{[58]}$ 、冰层厚度 ${ }^{[59]}$ 及冰雪覆盖程度等). 北半球历史资料 表明,在 1846-1995 年的 150 年间湖泊或者河流结冰时间平均每 100 年推迟 $5.8 \mathrm{~d}$,而冰融化时间开始提 前, 每 100 年约提前 $6.5 \mathrm{~d}^{[60]}$. 这些因素都能影响冰期水体中浮游植物的种类以及数量, 从而影响春季浮游 植物的演替 ${ }^{[61]}$.

另外, 全球变暖对浮游植物物候的影响还可能体现在累积效应——积温的增加. 积温的概念在昆虫和 高等植物研究中运用比较广泛, 在浮游植物研究中的运用还很少报道; 但藻类能快速增殖, 这种增殖可以看 做浮游植物对积温的一种响应, 并且在水温、营养盐和光照等条件适宜时浮游植物生物量将呈指数增长 ${ }^{[62]}$. 
以太湖为例, $\mathrm{Cao}$ 等 $^{[63]}$ 在太湖地区开展 的实验说明积温能更好地解释蓝藻复 苏过程. 在不同积温情境条件下, 水柱 中微囊藻生物量有差异但可能不显著, 常规采样误差甚至可能掩盖这种差异; 但是进人夏季 ( 水温超过 $20^{\circ} \mathrm{C}$ ), 微囊 藻开始指数增长, 上述微小的差异就能 被放大,从而使得夏季水华提前发生并 且程度加剧 (图 1).

\section{2 全球变暖对浮游植物群落结构的 影响}

浮游植物群落结构对全球变暖的 响应因季节、区域不同而有所差异. 同 时,不同物种对气候变化的响应也不相 同 $^{[65]}$,这是因为特定藻类对温度升高的 响应取决于该藻类的耐受范围与温度 变化范围之间的关系 ${ }^{[66]}$. 从 1960s 初开 始,对位于芬兰的 Pyhäjärvi 湖进行监 测, 结果表明, 平板藻 (Tabellaria) 、薄 甲藻 (Glenodinium) 、雉囊藻 (Dinobry-

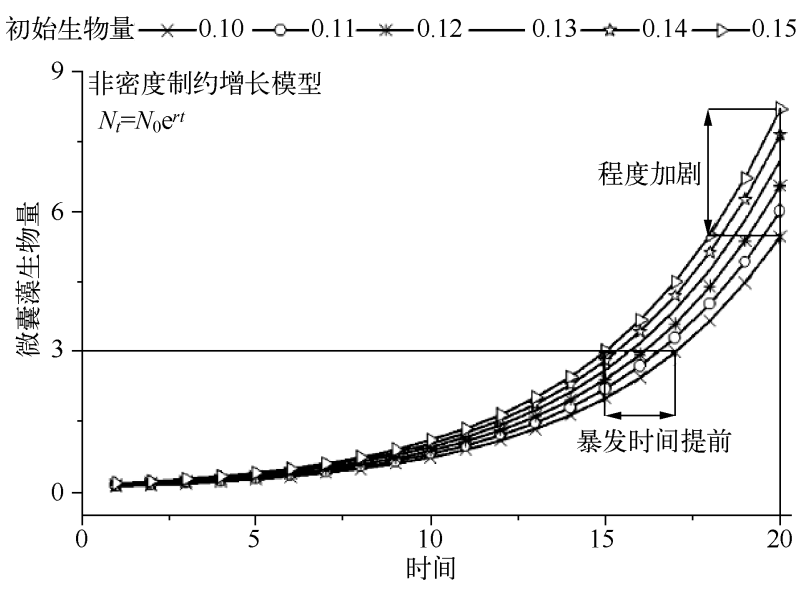

图 1 不同积温条件下前期不同累积微囊藻浓度对水华提 前暴发以及程度的影响 (初始生物量 $N_{0}$ 表示的是在不同 春季温度情景下, 水柱中可能累积的微囊藻生物量; 增长 速率 $(r)$ 为太湖野外原位所监测到的微囊藻实际增长速 率平均值 $^{[64]}, r=0.2 \mathrm{~d}^{-1}$ )

Fig. 1 Initial Microcystis biomass effects on blooms onset date and magnitudes $o n)$ 以及浮游蓝丝藻是该湖夏季主要优势种. Pyhäjärvi 湖夏季平均气温为 $17.97 \pm 1.28^{\circ} \mathrm{C}$, 薄甲藻最适生长 温度在 $24^{\circ} \mathrm{C}$ 左右 ${ }^{[67-68]}$; 雉囊藻最适生长温度范围为 $5 \sim 18^{\circ} \mathrm{C}{ }^{[69]}$; 浮游蓝丝藻最适生长温度范围为 $15 \sim$ $25^{\circ} \mathrm{C}^{[70]}$. 因为不同物种最适生长温度以及该湖夏季温度平均值的差异, 随着夏季温度的升高平板藻生物量 呈增加趋势, 但趋势并不显著 $(P>0.05)$; 而薄甲藻以及锥囊藻的生物量则逐渐下降; 浮游蓝丝藻生物量从 1990 年以来呈逐渐增加趋势, 2005 年以后成为绝对优势种, 即 Pyhäjärvi 湖地区的变暖趋势促进了浮游蓝丝 藻的优势、抑制了薄甲藻以及雉囊藻的优势, 而平板藻对全球变暖的响应似乎并不显著. Domis 等 ${ }^{[71]}$ 在 Tjeukemeer 湖 (位于荷兰北部) 开展的控制实验结果也表明, 在磷缺乏的情况下, 不同功能组的浮游植物对 气候变暖的响应是不一样的. Tahoe 湖是位于美国加利福尼亚州的一个深水湖泊, 1982-2006 年的监测数 据分析结果显示, 全球变暖引起的水柱热力学分层变化使得水柱中硅藻朝着小型硅藻演化, 因为与大型硅 藻相比,在稳定的水力分层情况下, 小型硅藻更具有竞争优势 (大型硅藻容易沉降 ${ }^{[72]}$.

总体来说, 在全球变暖的大背景下, 淡水湖泊中浮游植物群落结构正朝着蓝藻占优的方向发展 ${ }^{[73-74]}$. 首 先, 相对于硅藻和绿藻, 蓝藻喜高温 (往往 $>25^{\circ} \mathrm{C}$ ) ${ }^{[51]}$; 其次, 全球变暖使得春季提前, 秋季变冷推迟, 延长了 生长季节; 而且一般认为, 混合均匀的水体有利于硅藻 ${ }^{[75]}$ 、绿藻等易于沉降的种类生长, 而水体产生热力学 分层则有利于能自我调节浮力的蓝藻生 ${ }^{[76-77]}$; 同时, 全球变暖还将影响降雨格局, 使得流域内输人到水体 中的营养盐增加 ${ }^{[4,78]}$, 短时间内可能因为冲刷稀释作用使水华消失, 但是随着营养盐和水力停留时间增 加 ${ }^{[44]}$, 最终将促进蓝藻生长 ${ }^{[79]}$; 最后, 温度升高的同时营养盐浓度增加使得藻类大量生长, 也将降低湖水透 明度,使得某些能耐低光的蓝藻 (主要是颤藻目) 在光照较弱环境下获得竞争优势 ${ }^{[19]}$. 基于模型的研究结果 也表明水温增加对蓝藻优势的促进效果明显 ${ }^{[37]}$, 它使得水华发生提前 (温度上升 $1^{\circ} \mathrm{C}$ 时间提前 $2 \mathrm{~d}$ ) 并且蓝 藻所占比例也将增加 (温度上升 $1^{\circ} \mathrm{C}$ 藻所占比例增加 $7.6 \%$ ), 但是年平均生物量可能会下降 ${ }^{[35]}$, 这是因为当 春季生长受到促进时会大量吸收营养盐从而使营养盐成为限制性因子.

然而, 不同的研究认为水温升高对蓝藻的促进作用只有当营养盐达到一定阈值时才能体现出来 ${ }^{[80]}$, 其 中营养盐的作用可能更显著 ${ }^{[20]}$, 营养盐浓度、温度以及它们之间交互作用对蓝藻生长速率的影响可以用图 2 表示. Brookes 等 ${ }^{[81]}$ 认为营养盐对蓝藻生长的促进作用要大于水温, 因为随着营养盐浓度增加, 浮游植物 总生物量和蓝藻生物量也增加, 从而导致水下光场发生更强烈的光衰减, 影响水下短波的分布, 这使得水柱 


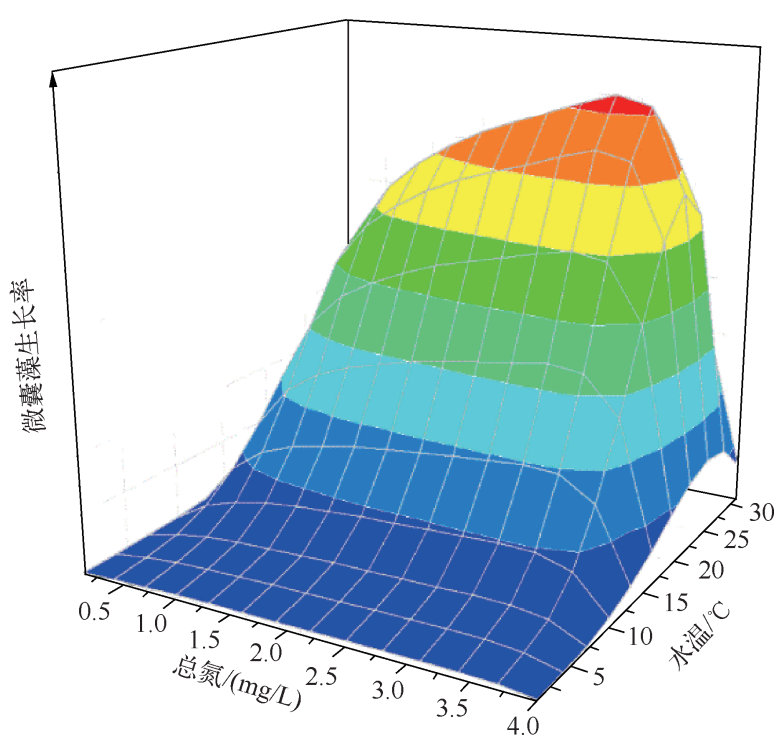

图 2 营养盐浓度和水温对微囊藻生长速率的共同影响 （最大生长速率与水温的关系参考文献 [ 85$]$, 与营养盐 的关系参考文献 $[86]$ )

Fig. 2 Interactive effects of nutrient concentrations and water temperature on Microcystis growth rate
能处于一个更加稳定的环境而进一步有 利于蓝藻占优 ${ }^{[82]}$. Wagner 等 ${ }^{[11]}$ 的研究 也有类似的结论, 认为在蓝藻发生的总 磷浓度阈值范围内 $(70 \sim 215 \mu \mathrm{g} / \mathrm{L})$, 气 候变暖才显著影响蓝藻的暴发概率, 而 这种促进的机理是因为气候变暖造成的 水体分层持续时间延长, 并不是水温本 身. 吴攀等 ${ }^{[83]}$ 在太湖地区开展的增温实 验结果表明, 营养盐对浮游植物群落结 构演替的贡献要大于水温, 而水温的升 高能使这种演替时间缩短、程度加剧. 同 样, 支持水温是促进蓝藻占优主要因子 的学者 Paerl 等 ${ }^{[84]}$ 和 Kosten 等 ${ }^{[19]}$ 也认为 由于温度升高的补偿作用, 水华发生对 营养盐浓度的需求可能会降低, 在全球 变暖背景下, 以前由于营养盐限制没有 发生水华的水体可能发生蓝藻水华.

对于亚热带 (太湖) 以及北温带湖泊 (Pyhäjärvi 湖) 中浮游植物群落演替的分 析表明, 随着全球变暖以及富营养化程 度加剧, 二者浮游植物群落结构都朝着 蓝藻占优的方向发展, 只是二者优势种

及机理不同. 太湖中的优势种并没有发生改变, 全球变暖更加强化了蓝藻的优势; 而 Pyhäjärvi 湖中浮游植物 优势种由原来的偏好低温种类 (锥囊藻等) 逐渐演变成现在的喜高温富营养化种类 (浮游蓝丝藻).

\section{3 全球变暖对浮游植物初级生产力的影响}

全球变暖对淡水浮游植物初级生产力的影响报道似乎不多 (且主要集中在北美) ${ }^{[65]}$, 最新的研究认为 全球变暖对初级生产力的影响通常取决于水体中的营养水平 ${ }^{[87]}$. 在营养盐浓度充足的情况下, 全球变暖可 能通过以下途径提高浮游植物的初级生产力. 首先, 全球变暖能延长生长季节; Byron 等 ${ }^{[88}$ 利用 $\mathrm{GCMs}$ ( General Circulation Models) 模型预测了 Castle 湖在 $\mathrm{CO}_{2}$ 浓度增加为 2 倍的情况下, 随着温度的增加、生长季节的 延长, 浮游植物年初级生产力亦增加. 另外, 同样在 Castle 湖中, 冬季降雪量大的年份生长季节开始晚, 来年 夏季初级生产力通常也较低 ${ }^{[89]}$. 另外两个可能的原因都与可利用的营养盐浓度有关. 第一个是水体热力学 分层的影响, 这在深水湖泊中尤为明显. 美国 Tahoe 湖的初级生产力主要受到春季最大混合深度的影响, 因 为春季水体混合是湖泊底部营养物质向上层水体迁移的主要过程 ${ }^{[89]}$. 同样在加拿大的湖泊中, 面积较大的 湖泊中初级生产力往往高于面积较小的湖泊,一方面较大的湖泊冰期较短, 另一方面是它们春季混合层深 度更大 ${ }^{[90]}$. 第二个原因是春季降雨量的影响, 在温带地区降雨量增加能携带更多的营养物质进人水 体 ${ }^{[4,78]}$, 从而提高夏季浮游植物初级生产力.

对 Geneva 湖历史数据分析的结果显示, 当磷的浓度充足时, 浮游植物的光合作用效率与水温呈正相 关 ${ }^{[87]}$. 但是当受到磷限制时, 光合作用效率与水温的关系会受到抑制; 随着磷的限制加强, 光合作用效率与 水温可能呈现负相关 ${ }^{[87]}$. 利用古湖沼学手段对贫营养湖泊 Tanganyika 湖的分析也发现, 在 1950-2000 年期 间, 磷的浓度非常低 (磷浓度平均约为 $8.99 \mu \mathrm{g} / \mathrm{L}$, 最低为 $0.31 \mu \mathrm{g} / \mathrm{L}$ ), 初级生产力在全球变暖大背景下 降低 ${ }^{[15,91]}$.

\section{3 展望}

1) 虽然各国科学家开展了大量的全球变暖对浮游植物影响的研究, 但由于资料以及研究方法等的限制 
以及由于多因子的共同作用 (如人类活动与全球变暖的耦合), 往往使得全球变暖对浮游植物群落的影响效 应复杂化,如何区分各因子的净影响份额是目前研究的一个难点; 更多历史资料的挖掘、更精细的实验设计 或许是将来突破的方向.

2) 我国湖泊大都为浅水湖泊, 底泥很容易受到风浪或者湖流的扰动而发生再悬浮 ${ }^{[92]}$, 底泥再悬浮也往 往伴随着营养盐的释放和水体悬浮物的大量增加 ${ }^{[93]}$; 对太湖的研究表明, 由于长江中下游水温偏高, 细菌活 性较强, 磷矿化速率非常快, 颗粒态磷最快能在几分钟内被碱性磷酸酶转化成溶解性反应磷供藻类利用 ${ }^{[94]}$. 气候变化引起风场的改变、影响再悬浮过程,造成水柱内营养盐的突变、水体浑浊而透明度下降等对浮游植 物的影响还未见报道. 同时, 国内目前还没有气候变化对流域内营养盐收支平衡影响的研究报道. 这些,都 可能成为我们新的研究切人点.

致谢: 吴揫、张玮以及马健荣对本文的修改提出了宝贵意见, Hans W. Paerl 对本文英文摘要进行了润色, 在 此一并致谢. 同时感谢审稿专家对本文改进提供的宝贵意见!

\section{4 参考文献}

[ 1 ] 张 强, 韩永翔, 宋连春. 全球气候变化及其影响因素研究进展综述. 地球科学进展, 2005, 20(9): 990-998.

[ 2 ] Mooij WM, Hülsmann S, Domis LNDS et al. The impact of climate change on lakes in the Netherlands: a review. Aquatic Ecology, 2005, 39(4) : 381-400.

[ 3 ] Nickus U, Bishop K, Erlandsson M et al. Direct impacts of climate change on freshwater ecosystems. In: Kernan MR, Battarbee RW, Moss B eds. Climate change impacts on freshwater ecosystems. UK: Blackwell Publishing Ltd, 2010: $38-64$.

[ 4 ] Jeppesen E, Kronvang B, Olesen JE et al. Climate change effects on nitrogen loading from cultivated catchments in Europe: implications for nitrogen retention, ecological state of lakes and adaptation. Hydrobiologia, 2011, 663(1) : 1-21.

[ 5 ] Rustad L, Campbell J, Marion G et al. A meta-analysis of the response of soil respiration, net nitrogen mineralization, and aboveground plant growth to experimental ecosystem warming. Oecologia, 2001, 126(4) : 543-562.

[ 6 ] Jack Brookshire EN, Gerber S, Webster JR et al. Direct effects of temperature on forest nitrogen cycling revealed through analysis of long-term watershed records. Global Change Biology, 2011, 17(1) : 297-308.

[ 7 ] Moss B, Kosten S, Meerhof M et al. Allied attack: climate change and eutrophication. Inland Waters, 2011, 1(2): 101-105.

[ 8 ] Hellmann JJ, Byers JE, Bierwagen BG et al. Five potential consequences of climate change for invasive species. Conservation Biology, 2008, 22(3) : 534-543

[ 9 ] Thompson R, Clark R. Is spring starting earlier? The Holocene, 2008, 18(1) : 95-104.

[10] Adrian R, O'Reilly CM, Zagarese H et al. Lakes as sentinels of climate change. Limnology and Oceanography, $2009, \mathbf{5 4}$ (6) : 2283-2297.

[11] Wagner C, Adrian R. Cyanobacteria dominance: quantifying the effects of climate change. Limnology and Oceanography, $2009, \mathbf{5 4}(6): 2460-2468$.

[12] Ventelä AM, Kirkkala T, Lendasse A et al. Climate-related challenges in long-term management of Säkylän Pyhäjärvi (SW Finland). Hydrobiologia, 2011, 660(1) : 49-58.

[13] Cai L, Zhu G, Zhu M et al. Effects of temperature and nutrients on phytoplankton biomass during bloom seasons in Taihu Lake. Water Science and Engineering, 2012, 5(4) : 361-374.

[14] Deng J, Qin B, Paerl HW et al. Earlier and warmer springs increase cyanobacterial (Microcystis spp. ) blooms in subtropical Lake Taihu, China. Freshwater Biology, 2014, 59(5) : 1076-1085.

[15] Verburg P, Hecky RE, Kling H. Ecological consequences of a century of warming in Lake Tanganyika. Science, 2003, $301(5632)$ : 505-507.

[16] Chen Y, Qin B, Teubner K et al. Long-term dynamics of phytoplankton assemblages: Microcystis-domination in Lake Taihu, a large shallow lake in China. Journal of Plankton Research, 2003, 25(4) : 445-453.

[17] Straile D, Jochimsen MC, Kümmerlin R. The use of long-term monitoring data for studies of planktonic diversity: a cautionary tale from two Swiss lakes. Freshwater Biology, 2013, 58(6) : 1292-1301.

[18 ] Jeppesen E, Meerhoff M, Davidson TA et al. Climate change impacts on lakes: an integrated ecological perspective based on a multi-faceted approach, with special focus on shallow lakes. Journal of Limnology, 2014, 73(s1) : 88-111.

[19] Kosten S, Huszar VLM, Bécares E et al. Warmer climate boosts cyanobacterial dominance in shallow lakes. Global Change Biology, 2012, 18(1) : 118-126.

[20] Rigosi A, Carey CC, Ibelings BW et al. The interaction between climate warming and eutrophication to promote cyanobac- 
teria is dependent on trophic state and varies among taxa. Limnology and Oceanography, 2014, 59: 99-114.

[21] Ekstrand S. Landsat TM based quantification of chlorophyll-a during algae blooms in coastal waters. International Journal of Remote Sensing , 1992, 13(10) : 1913-1926.

[22] Yacobi YZ, Gitelson A, Mayo M. Remote sensing of chlorophyll in Lake Kinneret using highspectral-resolution radiometer and Landsat TM: spectral features of reflectance and algorithm development. Journal of Plankton Research, 1995, 17 (11) : 2155-2173.

[23] Duan H, Ma R, Xu X et al. Two-decade reconstruction of algal blooms in China's Lake Taihu. Environmental Science and Technology, 2009, 43(10): 3522-3528.

[24] Zhang M, Duan HT, Shi XL et al. Contributions of meteorology to the phenology of cyanobacterial blooms: implications for future climate change. Water Research, 2012, 46(2) : 442-452.

[25] McKee D, Atkinson D, Collings S et al. Heated aquatic microcosms for climate change experiments. Freshwater Forum, $2000,14: 51-58$.

[26] Moss B, McKee D, Atkinson D et al. How important is climate? Effects of warming, nutrient addition and fish on phytoplankton in shallow lake microcosms. Journal of Applied Ecology, 2003, 40(5) : 782-792.

[27 Liboriussen L, Landkildehus F, Meerhoff M et al. Global warming: Design of a flow-through shallow lake mesocosm climate experiment. Limnology and Oceanography: Methods, 2005,3 : 1-9.

[28 ] Yvon-Durocher G, Jones JI, Trimmer M et al. Warming alters the metabolic balance of ecosystems. Philosophical Transactions of the Royal Society B: Biological Sciences, 2010, 365(1549) : 2117-2126.

[29] de Senerpont Domis LN, Mooij WM, Huisman J. Climate-induced shifts in an experimental phytoplankton community : a mechanistic approach. Hydrobiologia, 2007, 584(1): 403-413.

[30] Zhang M, Yu Y, Yang Z et al. Photochemical responses of phytoplankton to rapid increasing-temperature process. Phycological Research, 2012, 60(3) : 199-207.

[31] Thompson RM, Beardall J, Beringer J et al. Means and extremes: building variability into community-level climate change experiments. Ecology Letters, 2013, 16(6) : 799-806.

[32] Stewart RI, Dossena M, Bohan DA et al. Mesocosm experiments as a tool for ecological climate-change research. Advances in Ecological Research, 2013, 48: 71-181.

[33] Elliott JA. Is the future blue-green? A review of the current model predictions of how climate change could affect pelagic freshwater cyanobacteria. Water Research, 2012, 46(5) : 1364-1371.

[34] Howard A, Easthope MP. Application of a model to predict cyanobacterial growth patterns in response to climatic change at Farmoor Reservoir, Oxfordshire, UK. The Science of the Total Environment, 2002, 282 : 459-469.

[35] Elliott J, Thackeray SJ, Huntingford C et al. Combining a regional climate model with a phytoplankton community model to predict future changes in phytoplankton in lakes. Freshwater Biology, 2005, 50(8) : 1404-1411.

[36] Arheimer B, Andréasson J, Fogelberg S et al. Climate change impact on water quality: model results from southern Sweden. AMBIO: A Journal of the Human Environment, 2005, 34(7) : 559-566.

[37] Elliott J, Jones I, Thackeray S. Testing the sensitivity of phytoplankton communities to changes in water temperature and nutrient load, in a temperate lake. Hydrobiologia, 2006, 559(1) : 401-411.

[38 ] Mooij WM, Trolle D, Jeppesen E et al. Challenges and opportunities for integrating lake ecosystem modelling approaches. Aquatic Ecology, 2010, 44(3) : 633-667.

[39] Nielsen A. Predicting the future state of freshwater lake ecosystems influencedby climate and land use changes. Aarhus: Aarhus University, 2013.

[40 ] Kernan M, Battarbee R, Moss B. Changing climate and changing freshwaters: A European perspective. London: Blackwell, 2010.

[41] Gregory-Eaves I, Beisner BE. Palaeolimnological insights for biodiversity science: an emerging field. Freshwater Biology, $2011, \mathbf{5 6}(12):$ 2653-2661.

[42] Dong XH, Bennion H, Maberly SC et al. Nutrients exert a stronger control than climate on recent diatom communities in Esthwaite Water: evidence from monitoring and palaeolimnological records. Freshwater Biology, 2012, 57 (10): 2044-2056.

[43] Thies H, Tolotti M, Nickus U et al. Interactions of temperature and nutrient changes: effects on phytoplankton in the Piburger See (Tyrol, Austria). Freshwater Biology, 2012, 57 (10) : 2057-2075.

[44] Meis S, Thackeray SJ, Jones ID. Effects of recent climate change on phytoplankton phenology in a temperate lake. Fresh- 
water Biology, 2009, 54(9) : 1888-1898.

[45] Menzel A, Sparks TH, Estrella N et al. European phenological response to climate change matches the warming pattern. Global Change Biology, 2006, 12(10): 1969-1976.

[46] Parmesan C, Yohe G. A globally coherent fingerprint of climate change impacts across natural systems. Nature, 2003,421 (6918) : 37-42.

[47] Parmesan C. Influences of species, latitudes and methodologies on estimates of phenological response to global warming. Global Change Biology, 2007, 13(9): 1860-1872.

[48] Gerten D, Adrian R. Climate-driven changes in spring plankton dynamics and the sensitivity of shallow polymictic lakes to the North Atlantic Oscillation. Limnology and Oceanography, 2000, 45 (5) : 1058-1066.

[49] Sommer U, Lengfellner K. Climate change and the timing, magnitude, and composition of the phytoplankton spring bloom. Global Change Biology, 2008, 14(6) : 1199-1208.

[50] Patrick R. The effects of increasing light and temperature on the structure of diatom communities. Limnology and Oceanography, 1971, 16: 405-421.

[51] Reynolds CS. The ecology of phytoplankton. London: Cambridge University Press, 2006.

[52] Reynolds CS. The ecology of freshwater phytoplankton. London: Cambridge University Press, 1984.

[53] Winder M, Schindler DE. Climate change uncouples trophic interactions in an aquatic ecosystem. Ecology, 2004, 85(8) : 2100-2106.

[54] Thackeray S, Jones I, Maberly S. Long-term change in the phenology of spring phytoplankton: species-specific responses to nutrient enrichment and climatic change. Journal of Ecology, 2008, 96(3) : 523-535.

[55] Winder M. Limnology: Lake warming mimics fertilization. Nature Climate Change, 2012, 2(11) : 771-772.

[56] Winder M, Schindler DE. Climatic effects on the phenology of lake processes. Global Change Biology, 2004, 10 (11): 1844-1856.

[57] Wright RT. Dynamics of a phytoplankton community in an ice-covered lake. Limnology and Oceanography, 1964, 9(2): $163-178$

[58] Latifovic R, Pouliot D. Analysis of climate change impacts on lake ice phenology in Canada using the historical satellite data record. Remote Sensing of Environment, 2007, 106 (4) : 492-507.

[59] Todd MC, Mackay AW. Large-scale climatic controls on Lake Baikal ice cover. Journal of Climate, 2003, 16 (19) : 31863199.

[60 ] Magnuson JJ, Robertson DM, Benson BJ et al. Historical trends in lake and river ice cover in the Northern Hemisphere. Science, 2000, 289(5485) : 1743-1746.

[61] Adrian R, Walz N, Hintze T et al. Effects of ice duration on plankton succession during spring in a shallow polymictic lake. Freshwater Biology, 1999, 41(3) : 621-634.

[62] Reynolds CS. Physical determinants of phytoplankton succession. In: Plankton ecology. Berlin: Springer, 1989: 9-56.

[63] Cao HS, Tao Y, Kong FX et al. Relationship between temperature and cyanobacterial recruitment from sediments in laboratory and field studies. Journal of Freshwater Ecology, 2008, 23(3) : 405-412.

[64] 吴晓东, 孔繁翔. 水华期间太湖梅梁湾微囊藻原位生长速率的测定. 中国环境科学, 2008, 28(6): 552-555.

[65] Gerten D, Adrian R. Effects ofclimate warming, North Atlantic Oscillation, and El Niño-Southern Oscillation on thermal conditions and plankton dynamics in northern hemispheric lakes. The Scientific World Journal, 2002, 2 : 586-606.

[66] Walther GR. Adaptedbehaviour and shifting species ranges of species-a result of recent cliamte warming. In: Walther GR, Burga CA, Edwards PJ eds. "Fingerprints” of climate change: Adapted behaviour and shifting species ranges. New York: Kluwer Academic/Plenum Publishers, 2001.

[67] Bravo I, Anderson DM. The effects of temperature, growth medium and darkness on excystment and growth of the toxic dinoflagellate Gymnodinium catenatum from northwest Spain. Journal of Plankton Research, 1994, 16(5) : 513-525.

[68] Nielsen MV. Growth and chemical composition of the toxic dinoflagellate Gymnodinium galatheanum in relation to irradiance, temperature and salinity. Marine Ecology Progress Series, 1996, 136(1) : 205-211.

[69] Heinze AW. The role of temperature in the distribution of mixotrophic protists of the genus Dinobryon [Dissertation]. Philadelphia: Temple University, 2009

[70] Oberhaus L, Briand J, Leboulanger C et al. Comparative effects of the quality and quantity of light and temperature on the growth of Planktothrix agardhii and P. rubescens. Journal of Phycology, 2007, 43(6) : 1191-1199.

[71] Domis LNDS, Mooij WM, Huisman J. Climate-induced shifts in an experimental phytoplankton community: a mechanistic 
approach. Hydrobiologia, 2007, 584 (1) : 403-413.

[72] Winder M, Reuter JE, Schladow SG. Lake warming favours small-sized planktonic diatom species. Proceedings of the Royal Society B: Biological Sciences, 2009, 276(1656) : 427-435.

[73] Paerl HW, Huisman J. Blooms like it hot. Science, 2008, 320(5872) : 57-58.

[74] Carey CC, Ibelings BW, Hoffmann EP et al. Eco-physiological adaptations that favour freshwater cyanobacteria in a changing climate. Water Research, 2012, 46(5) : 1394-1407.

[75] Huisman J, van Oostveen P, Weissing FJ. Critical depth and critical turbulence: two different mechanisms for the development of phytoplankton blooms. Limnology and Oceanography, 1999, 44(7) : 1781-1787.

[76] Huisman J, Sharples J, Stroom JM et al. Changes in turbulent mixing shift competition for light between phytoplankton species. Ecology, 2004, 85(11): 2960-2970.

[77] Posch T, Köster O, Salcher MM et al. Harmful filamentous cyanobacteria favoured by reduced water turnover with lake warming. Nature Climate Change, 2012, 2 (11) : 809-813.

[78 ] Jeppesen E, Kronvang B, Meerhoff M et al. Climate change effects on runoff, catchment phosphorus loading and lake ecological state, and potential adaptations. Journal of Environmental Quality, 2009, 38(5) : 1930-1941.

[79] Elliott J. The seasonal sensitivity of cyanobacteria and other phytoplankton to changes in flushing rate and water temperature. Global Change Biology, 2010, 16(2) : 864-876.

[80] Mooij WM, Janse J, Domis LDS et al. Predicting the effect of climate change on temperate shallow lakes with the ecosystem model PCLake. In: de Pauw N ed. Shallow lakes in a changing world. Dordrecht: Springer Netherlands, 2007: 443454.

[81] Brookes JD, Carey CC. Resilience to blooms. Science, 2011, 334(6052) : 46-47.

[82] Kumagai M, Nakano S, Jiao C et al. Effect of cyanobacterial blooms on thermal stratification. Limnology, 2000, 1(3): 191-195.

[83] 吴 攀, 邓建明, 秦伯强等. 水温和营养盐增加对太湖冬, 春季节藻类生长的影响. 环境科学研究, 2013,26 (10) : 1064-1071.

[84] Paerl HW, Paul VJ. Climate change: links to global expansion of harmful cyanobacteria. Water Research, 2012, 46(5): 1349-1363.

[85] Jöhnk KD, Huisman J, Sharples J et al. Summer heatwaves promote blooms of harmful cyanobacteria. Global Change Biology, 2008, 14(3): 495-512.

[86] Xu H, Paerl HW, Qin B et al. Nitrogen and phosphorus inputs control phytoplankton growth in eutrophic Lake Taihu, China. Limnology and Oceanography, 2010, 55(1): 420-432.

[87] Tadonléké RD. Evidence of warming effects on phytoplankton productivity rates and their dependence on eutrophication status. Limnology and Oceanography, 2010, 55(3): 973.

[88 ] Byron ER, Goldman CR. The potential effects of global warming on the primary productivity of a subalpine lake. Journal of the American Water Resources Association, 1990, 26 (6) : 983-989.

[89] C'harles RG, Jassby A, Powell T. Interannual fluctuations in primary production: meteorological forcing at two subalpine lakes. Limnology and Oceanography, 1989, 34(2) : 310-323.

[90] Fee E, Shearer J, DeBruyn E et al. Effects of lake size on phytoplankton photosynthesis. Canadian Journal of Fisheries and Aquatic Sciences, 1992, 49(12) : 2445-2459.

[91] O'Reilly CM, Alin SR, Plisnier PD et al. Climate change decreases aquatic ecosystem productivity of Lake Tanganyika, Africa. Nature, 2003, 424(6950): 766-768.

[92] 秦伯强, 胡维平, 高 光等. 太湖沉积物悬浮的动力机制及内源释放的概念性模式. 科学通报, 2003, 48(17): 1822-1831.

[93] 朱广伟, 秦伯强, 张 路等. 太湖底泥悬浮中营养盐释放的波浪水槽试验. 湖泊科学, 2005, 17(1): 61-68.

[94] 高 光, 朱广伟, 秦伯强等. 太湖水体中碱性磷酸酶的活性及磷的矿化速率. 中国科学: D 辑, 2005, 35 (增刊 II ) : 157-165. 